\title{
AN INCLUSION THEOREM FOR MODULAR GROUPS ${ }^{1}$
}

\author{
MORRIS NEWMAN
}

Let $G$ denote the multiplicative group of $2 \times 2$ matrices

$$
\left(\begin{array}{ll}
a & b \\
c & d
\end{array}\right)
$$

where $a, b, c, d$ are rational integers and $a d-b c=1$. Let $G(m, n)$ denote the subgroup of $G$ characterized by $b \equiv 0(\bmod m)$ and $c \equiv 0$ $(\bmod n)$, where $m$ and $n$ are nonzero rational integers. In a previous paper [1] the author has proved Theorem I below:

Theorem I. Let $H$ be a subgroup of $G$ containing $G(1, n)$. Then $H=G\left(1, n_{1}\right)$, where $n_{1} \mid n$.

More generally, let $R$ be the ring of algebraic integers in a fixed algebraic number field of finite degree over the rationals. Let $G_{R}$ denote the multiplicative group of $2 \times 2$ matrices

$$
\left(\begin{array}{ll}
\alpha & \beta \\
\gamma & \delta
\end{array}\right),
$$

where $\alpha, \beta, \gamma, \delta$ are elements of $R$ and $\alpha \delta-\beta \gamma=1$. Let $G_{R}(\mathfrak{m}, \mathfrak{n})$ denote the subgroup of $G_{R}$ characterized by $\beta \in \mathfrak{m}$ and $\gamma \in \mathfrak{n}$, where $\mathfrak{m}$ and $\mathfrak{n}$ are nonzero ideals in $R$. Then Theorem I has been generalized by Reiner and Swift in a forthcoming paper [2] in the following manner:

Theorem II. Suppose that $(\mathfrak{n},(6))=(1)$, and let $H$ be a subgroup of $G_{R}$ containing $G_{R}((1), \mathfrak{n})$. Then $H=G_{R}\left((1), \mathfrak{n}_{1}\right)$, where $\mathfrak{n}_{1}$ is an ideal dividing $\mathfrak{n}$.

The restriction that $\mathfrak{n}$ be prime to (6) is necessary in general, examples being given in [2] which show that Theorem II may be false otherwise.

We propose to prove here the following generalizations of Theorems I and II:

TheOREM 1. Suppose that $(m, n)=1$. Let $H$ be a subgroup of $G$ containing $G(m, n)$. Then $H=G\left(m_{1}, n_{1}\right)$, where $m_{1} \mid m$ and $n_{1} \mid n$.

Theorem 2. Suppose that $(\mathfrak{m},(6))=(\mathfrak{n},(6))=(\mathfrak{m}, \mathfrak{n})=(1)$. Let $H$ be

Received by the editors September 16, 1953.

1 The preparation of this paper was supported (in part) by the Office of Naval Research. 
a subgroup of $G_{R}$ containing $G_{R}(\mathfrak{m}, \mathfrak{n})$. Then $H=G_{R}\left(\mathfrak{m}_{1}, \mathfrak{n}_{1}\right)$, where $\mathfrak{m}_{1}$ and $\mathfrak{n}_{1}$ are ideals dividing $\mathfrak{m}$ and $\mathfrak{n}$ respectively.

The restriction that $(m, n)=1$ (or that $(\mathfrak{m}, \mathfrak{n})=(1))$ is not superfluous. We prove as a companion theorem to these theorems the following:

THEOREM 3. Suppose that $(m, n)=k>1$. Then there are subgroups of $G$ containing $G(m, n)$ which are not of the form $G\left(m_{1}, n_{1}\right)$ where $m_{1} \mid m$ and $n_{1} \mid n$.

Theorem 3 of course applies to both Theorems 1 and 2 .

The proofs of Theorems 1 and 2 are not different, and we give only the proof of Theorem 2.

Since $(\mathfrak{m}, \mathfrak{n})=(1)$, there is an element $\mu$ of $\mathfrak{m}$ and an element $\nu$ of $\mathfrak{n}$ such that $\mu-\nu=1$. Thus the matrix

$$
\mathrm{r}=\left(\begin{array}{ll}
\mu & 1 \\
\nu & 1
\end{array}\right)
$$

is an element of $G_{R}$.

Suppose now that

$$
A=\left(\begin{array}{ll}
\alpha & \beta \\
\gamma & \delta
\end{array}\right) \in G_{R}(\mathfrak{m}, \mathfrak{n})
$$

Then the element in the $(2,1)$ place of $K^{-1} A K$ is $\mu \nu \delta-\mu \nu \alpha+\mu^{2} \gamma-\nu^{2} \beta$, and so $K^{-1} A K \in G_{R}((1), \mathfrak{m n})$ since $\mu \nu, \mu \gamma$, and $\nu \beta$ are all elements of $\mathfrak{m n}$. Thus $K^{-1} G_{R}(\mathfrak{m}, \mathfrak{n}) K \subseteq G_{R}((1), \mathfrak{m n})$.

Similarly, if $A \in G_{R}((1), \mathfrak{m n})$, we can show that $K A K^{-1} \in G_{R}(\mathfrak{m}, \mathfrak{n})$, which implies that $K G_{R}((1), \mathfrak{m n}) K^{-1} \subseteq G_{R}(\mathfrak{m}, \mathfrak{n})$, so that $K^{-1} G_{R}(\mathfrak{m}$, $\mathfrak{n}) K \supseteq G_{R}((1), \mathfrak{m n})$. This together with the preceding relationship proves that $K^{-1} G_{R}(\mathfrak{m}, \mathfrak{n}) K=G_{R}((1), \mathfrak{m n})$. In this manner we can show that for the same $K$

(1) If the ideals $\mathfrak{m}_{1}, \mathfrak{n}_{1}$ are any divisors of the ideals $\mathfrak{m}, \mathfrak{n}$ respectively, then $K^{-1} G_{R}\left(\mathfrak{m}_{1}, \mathfrak{n}_{1}\right) K=G_{R}\left((1), \mathfrak{m}_{1} \mathfrak{n}_{1}\right)$.

Suppose now that $H$ is a group such that

$$
G_{R}(\mathfrak{m}, \mathfrak{n}) \subseteq H \subseteq G_{R} .
$$

Then

$$
K^{-1} G_{R}(\mathfrak{m}, \mathfrak{n}) K \subseteq K^{-1} H K \subseteq K^{-1} G_{R} K .
$$

Using (1), we have

$$
G_{R}((1), \mathfrak{m}, \mathfrak{n}) \subseteq K^{-1} H K \subseteq G_{R} .
$$


Since $K^{-1} H K$ is a subgroup of $G_{R}$, and $(\mathfrak{m n},(6))=(1)$, Theorem II applies and we find that $K^{-1} H K=G_{R}((1), \mathfrak{l})$, where $\mathfrak{l} \mid \mathfrak{m n}$. Since $(m, n)=(1)$, we have $\mathfrak{l}=\mathfrak{m}_{1} \mathfrak{n}_{1}$, where $\mathfrak{m}_{1}\left|\mathfrak{m}, \mathfrak{n}_{1}\right| \mathfrak{n}$. Using (1) once again we find that $H=K G_{R}\left((1), \mathfrak{m}_{1} \mathfrak{n}_{1}\right) K^{-1}=G_{R}\left(\mathfrak{m}_{1}, \mathfrak{n}_{1}\right)$. Theorem 2 is thus proved.

The only difference in the proof of Theorem 1 is that the restriction $(\mathfrak{m},(6))=(\mathfrak{n},(6))=(1)$ is unnecessary and that Theorem $I$ is used above, instead of Theorem II.

We turn now to Theorem 3 . We have that $(m, n)=k>1$. Let $p$ be any prime divisor of $k$, so that $G(p, p) \supseteq G(m, n)$. (Here and in what follows we use the fact that $G\left(m_{1}, n_{1}\right) \supseteq G(m, n)$ if and only if $m_{1} \mid m$, $\left.n_{1} \mid n\right)$. Let $T$ be the element

$$
\left(\begin{array}{rr}
0 & -1 \\
1 & 0
\end{array}\right)
$$

of $G$, and let $F$ be the smallest subgroup of $G$ containing $T$ and $G(p, p)$. Since $T^{2}=-I$ and $T$ commutes with $G(p, p), F$ consists of the totality $T^{\epsilon} G(p, p)$, where $\epsilon$ is 0 or 1 . Thus if

$$
A=\left(\begin{array}{ll}
a & b \\
c & d
\end{array}\right)
$$

is any element of $F$, either $b \equiv c \equiv 0(\bmod p)$ or $a \equiv d \equiv 0(\bmod p)$. We now note the following:

$$
\left(\begin{array}{ll}
1 & 1 \\
0 & 1
\end{array}\right) \text { is an element of } G(1, p) \text { but not of } F \text {. }
$$$$
\left(\begin{array}{ll}
1 & 0 \\
1 & 1
\end{array}\right) \text { is an element of } G(p, 1) \text { but not of } F \text {. }
$$

$F$ contains $G(p, p)$ properly, and is properly contained in $G$.

Thus $F$ is not any of the groups $G(1,1), G(1, p), G(p, 1), G(p, p)$. $F$ therefore is a group containing $G(m, n)$ which is not itself of the form $G\left(m_{1}, n_{1}\right)$ for any divisors $m_{1}, n_{1}$ of $m, n$ respectively and so furnishes an example for Theorem 3.

\section{REFERENCES}

1. M. Newman, Structure theorems for modular subgroups, Duke Math. J. vol. 22 (1955) pp. 25-32.

2. I. Reiner and J. D. Swift, Congruence subgroups of matrix groups, To appear in Pacific Journal of Math.

National Bureau of Standards 